\title{
Early Controlled Exercise and Timing of Treatment Following Concussion: A Critically Appraised Topic
}

\author{
Nicholas Hattrup, Hannah Gray, Mark Krumholtz, and Tamara C. Valovich McLeod
}

\begin{abstract}
Clinical Scenario: Recent systematic reviews have shown that extended rest may not be beneficial to patients following concussion. Furthermore, recent evidence has shown that patient with postconcussion syndrome benefit from an active rehabilitation program. There is currently a gap between the ability to draw conclusions to the use of aerobic exercise during the early stages of recovery along with the safety of these programs. Clinical Question: Following a concussion, does early controlled aerobic exercise, compared with either usual care or delayed exercise, improve recovery as defined by symptom duration and severity? Summary of Key Findings: After a thorough literature search, 5 studies relevant to the clinical question were selected. Of the 5 studies, 1 study was a randomized control trial, 2 studies were pilot randomized controlled trials, and 2 studies were retrospective. All 5 studies showed that implementing controlled aerobic exercise did not have an adverse effect on recovery. One study showed early aerobic exercise had a quicker return to school, and another showed a 2-day decrease in symptom duration. Clinical Bottom Line: There is sufficient evidence to suggest that early controlled aerobic exercise is safe following a concussion. Although early aerobic exercise may not always result in a decrease in symptom intensity and duration, it may help to improve the psychological state resulting from the social isolation of missing practices and school along with the cessation of exercise. Although treatments continue to be a major area of research following concussion, management should still consist of an interdisciplinary approach to individualized patient care. Strength of Recommendation: There is grade B evidence to support early controlled aerobic exercise may reduce the duration of symptoms following recovery while having little to no adverse events.
\end{abstract}

Keywords: brain concussion, rehabilitation, exercise, running

\section{Clinical Scenario}

The fifth international conference on concussion in sport recommends integrating supervised aerobic exercise early in the treatment phase and reducing periods of rest to only 24 to 48 hours after injury. ${ }^{1}$ Previous reasoning behind rest was that it reduced the risk for subsequent concussion ${ }^{2}$ and reduced potential stresses on the brain to promote recovery. ${ }^{3}$ However, recent systematic reviews ${ }^{4,5}$ determined that the extended rest may not be beneficial to patients, and moderate activity levels and early activity may help to decrease the risk of postconcussion syndrome and improve symptom duration. ${ }^{6}$ Furthermore, recent statements have proposed a framework for more active treatments targeting specific clusters of symptoms following concussion to decrease comorbidities and day-to-day symptoms. ${ }^{7-9}$

Current literature has noted the potential effectiveness of treatments following concussion; however, most of this literature has occurred in patients with postconcussion syndrome. ${ }^{10}$ Those patients who undergo a multimodel treatment protocol (aerobic exercise, imagery, agility exercises, and balance exercises $)^{11,12}$ or perform aerobic exercise alone have shown improvement compared with usual care and rest. ${ }^{13}$ There is currently a gap in the literature as moderate activity have been shown to be beneficial within the first few weeks following injury, ${ }^{5}$ although the treatment literature has only examined patients with symptoms for more than 4 weeks. ${ }^{10}$ Therefore, there is limited evidence to currently support

Hattrup, Gray, Krumholtz, and Valovich McLeod are with the Athletic Training Programs at A.T. Still University, Mesa, AZ. Valovich McLeod is also with the School of Osteopathic Medicine in Arizona, A.T. Still University, Mesa, AZ. Hattrup (sa199462@atsu.edu) is corresponding author. or refute the claim of early timing of active therapy following concussion and its potential benefits.

\section{Clinical Question}

Following a concussion, does early controlled aerobic exercise, compared with either usual care or delayed exercise, improve recovery as defined by symptom duration and severity?

\section{Summary of Search, Best Evidence Appraised, and Key Findings}

- Literature was searched for studies with level 3 evidence or higher that implemented early controlled aerobic exercise in the early phases of concussion management compared with usual care, rest during the early stage of recovery.

- The literature search returned 12 studies related to the clinical question; 5 studies met the inclusion criteria.

- One study was a randomized controlled trial showing aerobic exercise compared to a stretching routine resulted in a 2-day decrease in symptoms. ${ }^{14}$

- One article ${ }^{15}$ demonstrated that initiating aerobic exercise had a positive effect on returning to school/work and sport sooner rather than delayed exercise.

- All 5 articles ${ }^{14-18}$ demonstrated that implementing early controlled aerobic exercise did not have a detrimental effect on recovery.

- One article ${ }^{17}$ demonstrated that although the early implementation of controlled aerobic exercise resulted in temporary increases in symptoms, it did not lengthen the overall duration of symptoms and the number of days to return-to-play. 
- Four articles investigated early controlled aerobic exercise as a treatment, ${ }^{16-18}$ whereas 1 article $^{18}$ investigated aerobic exercise as part of a multimodal treatment plan that also included manual therapy, strengthening activities, and ocular/vestibular exercise.

\section{Clinical Bottom Line}

There is sufficient evidence to suggest early controlled aerobic exercise is safe following a concussion but may not always be effective at reducing symptom intensity and duration. Although early exercise may help to reduce symptoms, this may not always lead to returning to play or sport in a shorter period. However, early aerobic exercise could potentially create a more positive experience for the patient by decreasing the affective symptoms potentially associated by being removed from sports participation along with isolation from peers. Furthermore, although early controlled aerobic exercise may be utilized as a treatment, each study had different treatment parameters (eg, duration and intensity) making it difficult to determine the optimal aerobic exercise protocol. Finally, although treatments continue to be on the forefront of concussion research and clinical practice, management should still include an interdisciplinary approach to individualized patient care.

\section{Strength of Recommendation}

There is grade B evidence to demonstrate early controlled aerobic exercise, either alone or in combination with a multimodal treatment plan, may reduce the duration of symptoms following recovery and appears to cause little to no adverse events.

\section{Search Strategy}

\section{Terms Used to Guide Search Strategy}

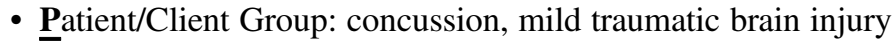

- Intervention (or Assessment): treatment, rehabilitation, aerobic exercise, and exercise

- Comparison: AND usual care AND rest

- Outcome(s): symptom, recovery, and duration

\section{Sources of Evidence Searched}

- The Cochrane Library

- CINAHL

- PubMed

- SPORTDiscus

- Hand searches was also performed

\section{Inclusion and Exclusion Criteria}

\section{Inclusion Criteria}

- Studies that examined implementation of early controlled aerobic exercise in recovery compared with usual care.

- Studies that examined the initiating of early controlled aerobic exercise in recovery and/or at various time points following the onset of a concussion.

- Studies utilizing interventions such as early controlled aerobic exercise, alone or part of a multimodal treatment, in the early weeks after injury (eg, <4 wk).

\section{Exclusion Criteria}

- Studies that investigated generic physical activity levels as a factor to recovery

- Studies that were published $>10$ years ago

- Studies that were below level 3 evidence

- Systematic reviews and abstracts

- Studies that only included 1 gender

\section{Results of Search}

Five relevant studies were located and categorized as shown in Table 1 (based on Levels of Evidence, Centre for Evidence Based Medicine, 2011).

\section{Best Evidence}

Table 2 describes the articles identified as best evidence and included for this critically appraised topic. The included studies were selected because they were considered level 3 evidence or higher and investigated the initiation of treatment/rehabilitation and compared early aerobic exercise with usual care.

\section{Implications for Practice, Education, and Future Research}

Currently, the fifth international conference on concussion in sport consensus statement encourages early treatment, such as early controlled aerobic exercise, following the first 48 hours of rest. ${ }^{1}$ Although there has been a growing amount of evidence for the effectiveness of controlled aerobic exercise to reduce persistent symptoms, the majority of included studies focused on patientinitiated activity or whether they followed recommendations rather than clinician programmed exercise or treatment. ${ }^{5}$ Based upon 2 articles included in this review, early controlled aerobic exercise initiated within 3 to 11 days postconcussion is safe but may not be effective at decreasing symptom duration and overall recovery. ${ }^{17,18}$ The other 3 studies demonstrated early controlled aerobic exercise from 3 to 6 days postinjury had a positive effect, ${ }^{14-16}$ meaning the earlier one started exercise the quicker the symptom resolution within the first 2 weeks. These findings may encourage a shift in current rehabilitation techniques from a conservative, rest-centered approach to a more active and progressive approach. Furthermore, an additional study not included in this synthesis due to its lower level of evidence, ${ }^{19}$ described a series of cases in which patients who underwent an active rehabilitation program, consisting of up

\section{Table 1 Summary of Study Designs of Articles Retrieved}

\begin{tabular}{|c|c|c|c|}
\hline $\begin{array}{l}\text { Level of } \\
\text { evidence }\end{array}$ & $\begin{array}{l}\text { Study } \\
\text { design }\end{array}$ & $\begin{array}{l}\text { Number } \\
\text { located }\end{array}$ & Author (year) \\
\hline 2 & $\begin{array}{l}\text { Randomized } \\
\text { controlled trial }\end{array}$ & 1 & Leddy et $\mathrm{al}^{14}$ (2019) \\
\hline 2 & $\begin{array}{l}\text { Pilot randomized } \\
\text { controlled trial }\end{array}$ & 2 & $\begin{array}{l}\text { Micay et al }{ }^{16} \text { (2018) } \\
\text { Maerlender et } \mathrm{al}^{17} \text { (2015) }\end{array}$ \\
\hline 3 & $\begin{array}{l}\text { Retrospective } \\
\text { cohort }\end{array}$ & 2 & $\begin{array}{l}\text { Lennon et } \mathrm{al}^{18}(2018) \\
\text { Lawrence et } \mathrm{al}^{15}(2018)\end{array}$ \\
\hline
\end{tabular}




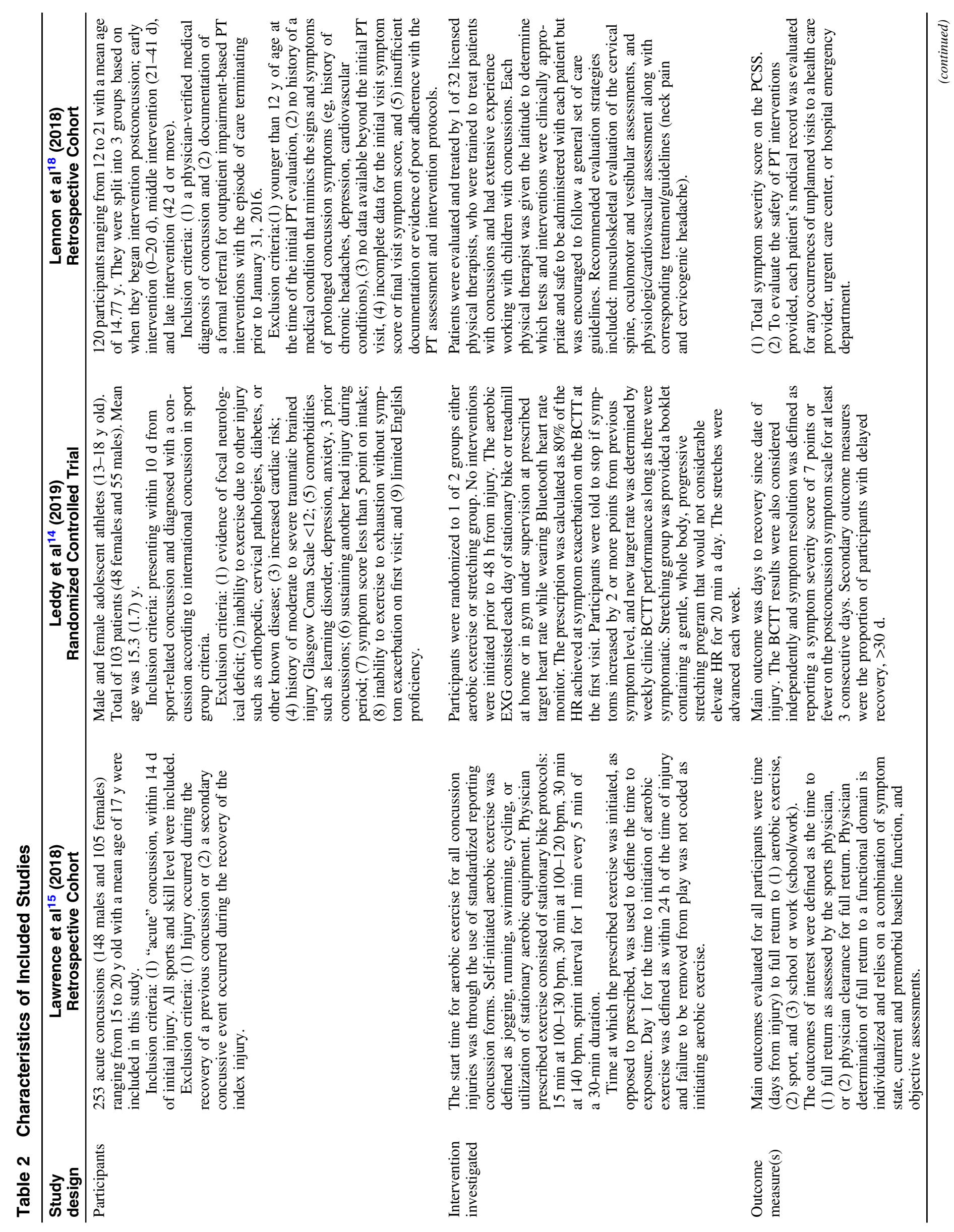




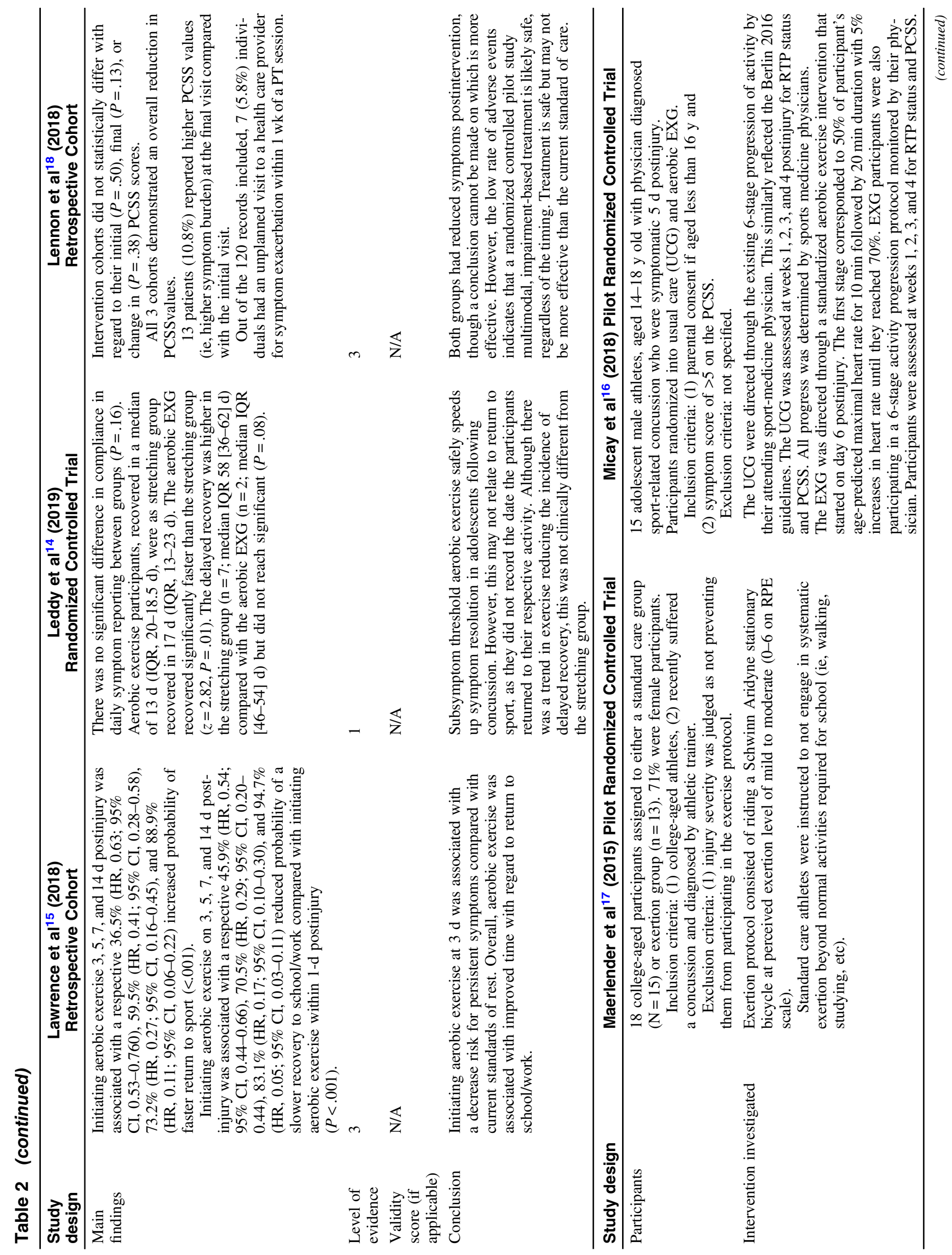




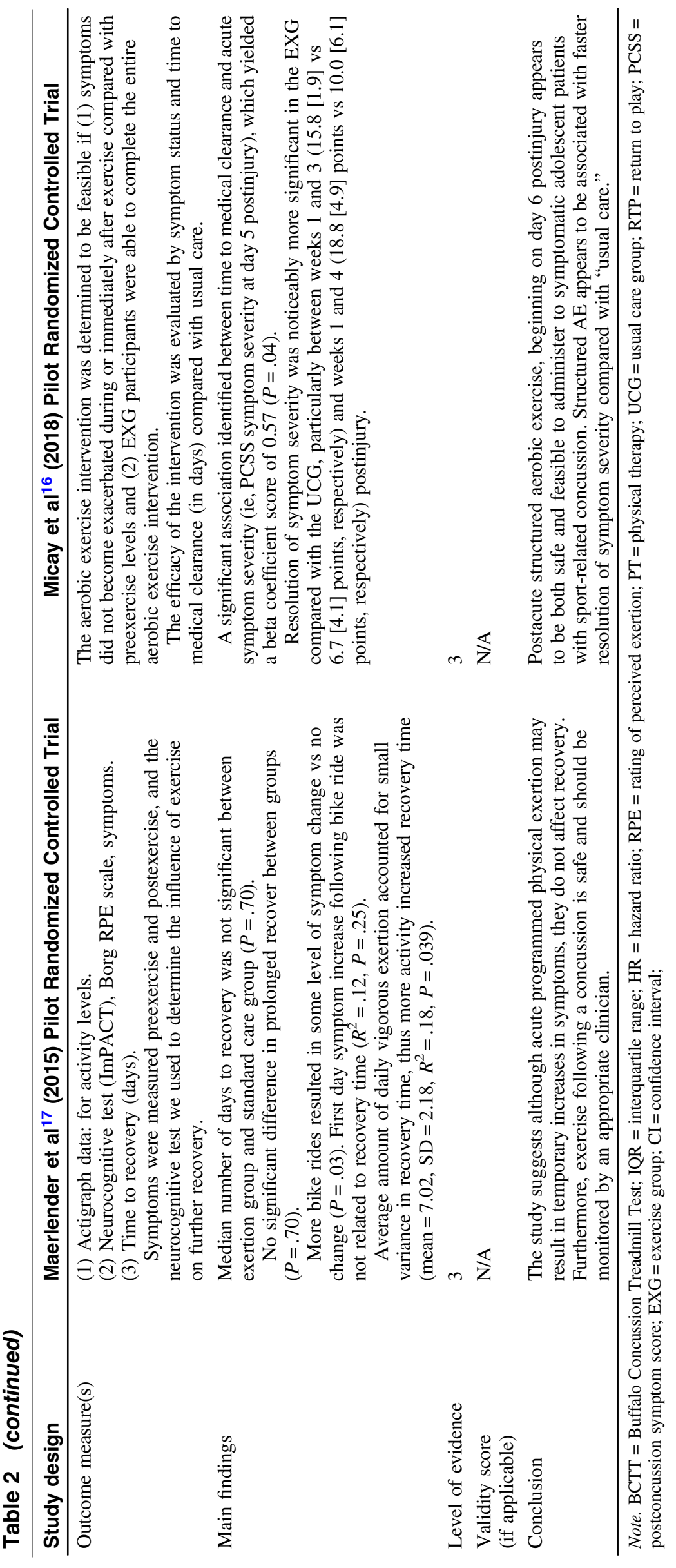


to 30 minutes of daily activity, before 2 weeks postinjury had a better improvement in symptom intensity and duration than those starting the program at 6 weeks or later.

Recent qualitative studies examining recovery have described the removal of patients following concussion from their daily social interactions along with the sudden decrease in aerobic fitness may negatively affect their quality of life. ${ }^{20,21}$ Early aerobic exercise, even while the patient is symptomatic, may help to decrease some of these sudden changes while improving the overall mood state of the individual ${ }^{22}$ and potentially reducing the risk of prolonged recovery. ${ }^{14}$ However, clinician supervision is essential in early controlled aerobic exercise to ensure the initiation of activity does not exacerbate symptoms and patients participate at a subsymptom threshold. ${ }^{17}$ The temporary increases in symptoms that may appear during or immediately following early aerobic exercise does not seem to hinder overall recovery timelines. Although the available research is not able to give definitive recommendations to change the standard of concussion management, the lack of adverse effects associated with early controlled exercises following a concussion is promising. To maintain safe exposure to exercise, clinicians should monitor patients and administer a progressive aerobic exercise program through appropriate supervision and documentation.

The use of bikes and treadmills were mostly used as the form of exercise across all studies. However, each study utilized slightly different methods to determine the appropriate intensity and duration for patients. Only one study ${ }^{14}$ utilized an exertional test (ie, Buffalo Concussion Treadmill Test) to determine specific heart rate ranges for each individual patient. Other studies utilized a general prescription of exercise based upon heart rate for all patients with increasing intensity at various stages, such as 15 minutes at 100 to $130 \mathrm{bpm}$ progressing to 30 minutes at 100 to $120 \mathrm{bpm},{ }^{15}$ whereas another study used an age-derived formula to prescribe a heart rate level. ${ }^{16}$ With each study prescribing varying intensities and frequencies of aerobic exercise, it is hard to determine the optimal level of early controlled aerobic exercise following a concussion. However, the collective literature seems to describe an exercise protocol beginning at a workload of 100 to $130 \mathrm{bpm}$ for a duration of 10 to 30 minutes, followed by a progression of increasing intensity and duration that remains below symptom thresholds. Overall, each patient should be supervised and treated with individualized care to provide treatment that is safe, timely, and effective.

Although most studies investigated programmed exercise as a sole treatment, 1 study examined multiple treatments consisting of impairment-targeted interventions, ${ }^{18}$ which more likely reflects what clinicians should do in practice. Utilizing this approach may be beneficial as symptoms experienced following a concussion are often clustered into concussion profiles or subtypes, including vestibular, ocular, migraine-headache, mood, or cervicogenic and each profile may require a specific type of treatment. ${ }^{8}$ The aerobic exercise program utilized in conjunction with the targeted treatments in this study was similar to the studies using only aerobic exercise and included symptom limited aerobic activity that increased in intensity over treatment sessions. Future research should determine if the addition of symptom targeted interventions in conjunction with early aerobic exercise has a more beneficial effect on symptom recovery.

Although some studies included in this critically appraised topic did not show a decreases in symptoms, ${ }^{17,18}$ early aerobic exercise appears to have a low risk for adverse avents. ${ }^{23}$ Furthermore, most patients demonstrate a desire to want to perform activity and be socially involved with their peers, school, and sport. ${ }^{24}$ After their recovery, patients identified that aerobic exercise and education regarding energy maintenance were the most useful and engaging parts of a concussion rehabilitation program. ${ }^{25}$ Therefore, even though early aerobic exercise may not always be better than usual care, it may increase the psychological state of the patient and improve the experiences during recovery. Future studies should continue to examine the role of treatment from both the patient's experience and recovery and from the clinician's perspective of comfort and resource utilization.

In conclusion, early treatment is safe with little risk for adverse event and may be effective at reducing symptoms following a concussion during the earlier stages, while providing psychosocial benefits to the patient. Although early aerobic exercise may be prudent, individualized clinical decision making considering each patients unique traits should be considered to ensure patient safety and monitor recovery. Furthermore, it is important to consider the potential positive effects each patient may have along with how it affects their experience and psychosomatic nature. Future research should continue to determine the best treatment following injury and correct dosage with regard to duration and frequency regarding early controlled aerobic exercise. This critically appraised topic should be reviewed in 2 years to update the current recommendation with newly available evidence.

\section{References}

1. McCrory P, Meeuwisse W, Dvorak J, et al. Consensus statement on concussion in sport - the 5th international conference on concussion in sport held in Berlin, October 2016. Br J Sports Med. 2017;51(11): 838-847. PubMed ID: 28446457

2. Saunders RL, Harbaugh RE. The second impact in catastrophic contact-sports head trauma. JAMA. 1984;252(4):538-539. PubMed ID: 6737652 doi:10.1001/jama.1984.03350040068030

3. Giza CC, Hovda DA. The new neurometabolic cascade of concussion. Neurosurgery. 2014;75(suppl 4):S24-S33. doi:10.1227/NEU. 0000000000000505

4. Lal A, Kolakowsky-Hayner SA, Ghajar J, Balamane M. The effect of physical exercise after a concussion: a systematic review and metaanalysis. Am J Sports Med. 2018;46(3):743-752. PubMed ID: 28570092 doi:10.1177/0363546517706137

5. McLeod TC, Lewis JH, Whelihan K, Bacon CE. Rest and return to activity after sport-related concussion: a systematic review of the literature. J Athl Train. 2017;52(3):262-287. PubMed ID: 28387547 doi:10.4085/1052-6050-51.6.06

6. Silverberg ND, Iverson GL. Is rest after concussion "the best medicine?": recommendations for activity resumption following concussion in athletes, civilians, and military service members. J Head Trauma Rehabil. 2013;28(4):250-259. PubMed ID: 22688215 doi:10. 1097/HTR.0b013e31825ad658

7. Craton N, Ali H, Lenoski S. COACH CV: the seven clinical phenotypes of concussion. Brain Sci. 2017;7(9):E119. PubMed ID: 28926944 doi:10.3390/brainsci7090119

8. Collins MW, Kontos AP, Reynolds E, Murawski CD, Fu FH. A comprehensive, targeted approach to the clinical care of athletes following sport-related concussion. Knee Surg Sports Traumatol Arthrosc. 2014;22(2):235-246. PubMed ID: 24337463 doi:10. 1007/s00167-013-2791-6

9. Ellis MJ, Leddy JJ, Willer B. Physiological, vestibulo-ocular and cervicogenic post-concussion disorders: an evidence-based classification system with directions for treatment. Brain Inj. 2015;29(2): 238-248. PubMed ID: 25314613 doi:10.3109/02699052.2014. 965207 
10. Schneider KJ, Leddy JJ, Guskiewicz KM, et al. Rest and treatment/ rehabilitation following sport-related concussion: a systematic review. Br J Sports Med. 2017;51(12):930-934. PubMed ID: 28341726 doi:10.1136/bjsports-2016-097475

11. Dobney DM, Grilli L, Kocilowicz H, et al. Evaluation of an active rehabilitation program for concussion management in children and adolescents. Brain Inj. 2017;31(13-14):1753-1759. doi:10.1080/ 02699052.2017.1346294

12. Gagnon I, Grilli L, Friedman D, Iverson GL. A pilot study of active rehabilitation for adolescents who are slow to recover from sportrelated concussion. Scand J Med Sci Sports. 2016;26(3):299-306. PubMed ID: 25735821 doi:10.1111/sms.12441

13. Leddy JJ, Kozlowski K, Donnelly JP, Pendergast DR, Epstein LH, Willer B. A preliminary study of subsymptom threshold exercise training for refractory post-concussion syndrome. Clin J Sport Med. 2010;20(1):21-27. PubMed ID: 20051730 doi:10.1097/JSM. 0b013e3181c6c22c

14. Leddy JJ, Haider MN, Ellis MJ, et al. Early subthreshold aerobic exercise for sport-related concussion: a randomized clinical trial [published online ahead of print February 4, 2019]. JAMA Pediatr. doi:10.1001/jamapediatrics.2018.4397

15. Lawrence DW, Richards D, Comper P, Hutchison MG. Earlier time to aerobic exercise is associated with faster recovery following acute sport concussion. PLoS ONE. 2018;13(4):e0196062. PubMed ID: 29668716 doi:10.1371/journal.pone.0196062

16. Micay R, Richards D, Hutchison MG. Feasibility of a postacute structured aerobic exercise intervention following sport concussion in symptomatic adolescents: a randomised controlled study. BMJ Open Sport Exerc Med. 2018;4(1):e000404. PubMed ID: 30018795 doi:10. 1136/bmjsem-2018-000404

17. Maerlender A, Rieman W, Lichtenstein J, Condiracci C. Programmed physical exertion in recovery from sports-related concussion: a randomized pilot study. Dev Neuropsychol. 2015;40(5):273-278. PubMed ID: 26230745 doi:10.1080/87565641.2015.1067706

18. Lennon A, Hugentobler JA, Sroka MC, et al. An exploration of the impact of initial timing of physical therapy on safety and outcomes after concussion in adolescents. J Neurol Phys Ther. 2018;42(3):123131. PubMed ID: 29846269 doi:10.1097/NPT.0000000000000227

19. Dobney DM, Grilli L, Kocilowicz H, et al. Is there an optimal time to initiate an active rehabilitation protocol for concussion management in children? A case series. J Head Trauma Rehabil. 2018;33(3):E11e17. PubMed ID: 28926482 doi:10.1097/HTR.0000000000000339

20. Valovich McLeod TC, Wagner AJ, Bacon CEW. Lived experiences of adolescent athletes following sport-related concussion. Orthop $J$ Sports Med. 2017;5(12):2325967117745033. PubMed ID: 29276716 doi: $10.1177 / 2325967117745033$

21. Iadevaia C, Roiger T, Zwart MB. Qualitative examination of adolescent health-related quality of life at 1 year postconcussion. $J$ Athl Train. 2015;50(11):1182-1189. PubMed ID: 26509684 doi:10.4085/ 1062-6050-50.11.02

22. Edwards MK, Loprinzi PD. Effects of a sedentary behavior-inducing randomized controlled intervention on depression and mood profile in active young adults. Mayo Clin Proc. 2016;91(8):984-998. PubMed ID: 27492908 doi:10.1016/j.mayocp.2016.03.021

23. Leddy JJ, Haider MN, Ellis M, Willer BS. Exercise is medicine for concussion. Curr Sports Med Rep. 2018;17(8):262-270. PubMed ID: 30095546 doi:10.1249/JSR.0000000000000505

24. Hunt TN, Harris LL. Psychological impact of cognitive rest following sport-related concussion on low socioeconomic status adolescent patients. J Allied Health. 2017;46(4):e81-e83. PubMed ID: 29202169

25. Hunt AW, Laupacis D, Kawaguchi E, Greenspoon D, Reed N. Key ingredients to an active rehabilitation programme post-concussion: perspectives of youth and parents. Brain Inj. 2018;32(12):1534 1540. PubMed ID: 30047796 doi:10.1080/02699052.2018.1502894 